\title{
De la gnose à la responsabilité
}

Hans Jonas, critique de l'acosmisme anthropologique

Jean-Philippe Pierron

\section{(2) OpenEdition}

Journals

Édition électronique

URL : http://journals.openedition.org/alter/301

DOI : 10.4000/alter.301

ISSN : 2558-7927

Éditeur :

Association ALTER, Archives Husserl (CNRS-UMR 8547)

Édition imprimée

Date de publication : 15 novembre 2014

Pagination : 145-162

ISBN : 978-2-9550449-0-2

ISSN : 1249-8947

Référence électronique

Jean-Philippe Pierron, « De la gnose à la responsabilité », Alter [En ligne], 22 | 2014, mis en ligne le 01 décembre 2017, consulté le 19 avril 2019. URL : http://journals.openedition.org/alter/301 ; DOI :

10.4000/alter.301

Ce document a été généré automatiquement le 19 avril 2019

Revue Alter 


\title{
De la gnose à la responsabilité
}

\author{
Hans Jonas, critique de l'acosmisme anthropologique
}

\section{Jean-Philippe Pierron}

L'homme est l'être qui tourmente la déesse auguste entre toutes, la Terre,

La Terre éternelle et infatigable, avec ses charrues

qui vont sans répit la sillonnant chaque année, celui qui la

fait labourer

par les produits de ses cavales. [...]

Mais, ainsi maître d'un savoir, dont les ingénieuses ressources dépassent toute

espérance,

il peut prendre ensuite la route du mal tout comme

du bien.

Sophocle, Antigone,

trad. P. Mazon, Paris, Les Belles Lettres, 1950, pp.

87-88.

1 Il est différentes expériences humaines de la nature. Il en est une, antique, qui l'exprime dans les mots d'un monde clos. L'homme y existe comme appartenant et soumis à l'ordre englobant d'une nature (physis) et à l'équilibre harmonieux d'un monde (cosmos) stable, sécurisant et finalisé. Il en est une autre qui en parle comme d'un univers infiniment étendu, celui d'une nature indifférente et nullement fraternelle. Soutenue par un dualisme entre une nature objet de connaissance et de maîtrise et un sujet humain qui se comprend comme étranger exilé dans une nature hostile, elle relève d'une sorte d'acosmisme anthropologique ${ }^{1}$. Il en est une autre enfin, qui tente de se libérer de cet acosmisme sans pour autant resacraliser la nature dans un cosmos. Elle se formule dans l'idée de Terre - signifiant commun fédérant les différentes options qu'engage la conscience écologique -, envisagée comme lieu d'habitation. S'y expérimentent une appartenance à une nature fraternelle et l'appel à une responsabilité singulière. De cette dernière option, Hans Jonas dira: «Savoir si une troisième voie s'offre à lui [l'esprit 
moderne], qui permettrait d'éviter la déchirure dualiste et de garder assez d'intuition dualiste pour maintenir l'humanité de l'homme, c'est la tâche de la philosophie $»^{2}$. Il en fera son programme: penser une conscience environnementale, soucieuse de tout être vulnérable, humain et non humain, non comme un antihumanisme mais comme un humanisme de l'autre homme.

Un enjeu fondamental est engagé dans la rénovation sémantique et pratique qui parle de la nature comme Terre ou biosphère comme préférera dire Jonas 3 . Philosophiquement, la Terre tente d'exprimer une appartenance originaire. Elle permet de penser les relations de l'homme avec la nature comme une dépendance sans hétéronomie. La ligne de crête est subtile. Nous avons du mal à la concevoir car nous pensons trop à une humanité qui soumet sa volonté à la nature et pas assez à une liberté qui s'ouvre en entendement et en imagination à une compréhension renouvelée de son appartenance au monde des vivants. Nous tendons à vivre la dépendance comme une humiliation de la dignité humaine et une contrariété de notre volonté de puissance. Or ne peut-on pas l'envisager comme un élargissement du sens donné à notre appartenance du côté de l'entendement et à une compréhension élargie en imagination de notre responsabilité vis-à-vis de l'histoire et de la nature vulnérable? Le projet philosophique de Hans Jonas assume ces deux volets. D'une part, il développe une herméneutique non tronquée de sciences du vivant trop souvent réduites à une physique du vivant. Il élabore une inédite biologie philosophique rompant avec le dualisme qui fait de la nature l'autre de l'homme. Confrontée à «l'appel muet du monde de la vie ", elle est ouverte à sa proposition de sens équivoque, posant que «les sciences de la nature ne livrent pas toute la vérité au sujet de la nature ${ }^{4}$. D'autre part, il explore les relations entre éthique et imagination dans une éthique du futur qui augmente la compréhension de ce qui nous solidarise avec les humains d'aujourd'hui et de demain mais également avec les non-humains. Mais en quoi la conscience environnementale serait-elle élargie en imagination dans une éthique du futur? Comment se trouve-t-elle instruite en entendement par la biologie philosophique? Entre l'objectivation acosmique de la nature et la cosmicité fusionnelle de l'orphisme, la perspective jonassienne parvient-elle à dépasser le dualisme homme/ nature et à rendre compte de l'entre-lien de l'homme et de la vie?

\section{1 - La philosophie de Hans Jonas et les éthiques de la nature}

Le mot «Terre » dans le vocabulaire phénoménologique enregistre un déplacement de la nature conçue comme sol à la nature comme corps. Rénovation sémantique d'un thème ancien - l'expérience humaine de l'univers - il atteste que le souci environnemental est lié à une expérience originaire à mettre au jour. Parler de Terre, c'est dire que le pays natal est moins une étendue qu'un milieu, moins une géométrie qu'une géo-pathie. Il est une géographie habitée à l'échelle globale. " En vertu de la fonction médiatrice du corps propre dans la structure de l'être dans le monde, le trait d'ipséité de la corporéité s'étend à celle du monde en tant que corporellement habité. Ce trait qualifie la condition terrestre en tant que telle et donne à la Terre la signification existentiale que, sous des guises diverses, Nietzsche, Husserl et Heidegger lui reconnaissent. La Terre est ici plus et autre chose qu'une planète. C'est le nom mythique de notre ancrage corporel dans le monde $»^{5}$. En amont d'une vérité de science fournie par les sciences écologiques, il est une vérité d'existence : être humain sur la Terre. 
Sans donner au concept de Terre la place attendue, lui préférant le phénomène de la Vie ou la biosphère, Hans Jonas a lui aussi installé sa réflexion d'éthique pour une civilisation technologique, hantée par la question du mal prométhéen ${ }^{6}$, dans une perspective existentiale. Il s'agit de penser l'appartenance de l'humain au monde des vivants. Mais il en a modifié la compréhension, pensant ensemble la conscience écologique d'une appartenance et l'exigence éthique d'une responsabilité. Il s'ensuit un certain nombre de conséquences. La première est que son éthique de la nature se distanciera d'une éthique de l'habiter attachée à l'épreuve d'un enracinement. La seconde se méfiera de l'éthique de l'arrachement propre à la civilisation technologique qui fait de l'homme un étranger sur la Terre. La troisième écartera une approche éthique à la manière des sagesses antiques développant des maximes pour bien vivre selon la nature telle qu'on en trouve aujourd'hui dans la recherche d'une frugalité volontaire portée par les mouvements alternatifs. La quatrième n'envisagera pas l'éthique de la nature comme une des sections de l'arbre de la philosophie, comme une discipline spécifique mais relèvera plutôt, rappelant Levinas, d'une éthique conçue comme philosophie première. Cela installe un étonnant décalage entre ce projet et celui des éthiques environnementales nordaméricaines. Alors que ces dernières s'attacheront à démontrer la valeur intrinsèque de la nature ou des non humains dans une éthique du respect, Jonas pensera les liens entre éthique et métaphysique, écologie et civilisation dans une éthique de la responsabilité.

Husserl ${ }^{7}$, mais surtout Heidegger, a trouvé dans l'expérience de l'habiter, faisant coïncider ethos et oikos, une disposition originaire nouant l'éthique à un habiter le là de notre être-là. ${ }^{8}$ Habiter rend compte de ce que signifie être humain sur la Terre, épreuve irréductible à la maîtrise des déterminations objectives. Habiter se concentre sur un sentir plus profond que le percevoir, attentif aux connivences sensibles, charnelles qui lient la nature et les hommes dans une poétique de la Terre. N'ignorant pas la méditation heideggérienne du "poétiquement l'homme habite le monde", mais se souvenant des analyses antérieures de Sein und Zeit consacrées à l'«avoir été jeté ", à la Geworfenheit (déjection), le projet de Hans Jonas rompt pourtant avec cette pensée de l'habiter. Il ne reprendra pas à son compte l'idéologie de l'enracinement, paradoxalement acosmique, dans le sol ou la Terre-natale qu'il véhicule, ni avant lui l'acosmisme existentiel. Sous la plume de Jonas, la biosphère, mieux que la Terre, sera le signifiant ontologique et éthique grâce auquel appréhender une nature conçue et vécue comme vulnérable dans un régime de coprésence et devenue nouvel objet de responsabilité. En ce sens, il complète, tout en se situant sur un autre plan discursif, l'idée de biosphère issue de la conjonction entre sciences de la terre et sciences de la vie, promue par le fondateur de l'écologie globale et inventeur du mot (1926), Wladimir Vernadsky9. Notablement, la biologie philosophique de Jonas, sans s'y confondre, se laissera instruire par les sciences du vivant. Là où une attitude phénoménologique cherchera du côté de l'esthétique au sens d'une expérience pathique originaire qui fait la différence entre être et y être (cf. Maldiney), le nœud renouant une appartenance de l'homme avec la Terre, Hans Jonas déploiera du côté d'une biologie philosophique corrigeant la biologie réduite à n'être qu'une physique du vivant une attention aux formes de vie engagées dans la végétalité, l'animalité ou l'humanité. Critiquant la science moderne de la nature qui exclut cette dernière du champ de la valeur, Hans Jonas écrit ainsi : « ne se manifeste-t-il pas là - pour passer de la description à la critique - le soupçon que l'image réduite d'une nature sans finalité - que la science s'est préparée en vue d'avoir une connaissance bien réfléchie faite pour un modèle de 
science défini - n'est cependant pas toute la vérité sur la nature, mais seulement une vue codifiée de manière artificielle $»^{10}$ ?

6 Attentif au phénomène de la vie alors qu'il est devenu convenu de dire qu'« on n'étudie pas la vie dans les laboratoires de biologie », Jonas consacre des descriptions à la vitalité du vivant et à sa fragilité, situées par-delà mécanisme et finalisme. L'ouverture du vivant au monde, qui caractérise sa dynamique relationnelle, risque, à chaque fois, de n'être plus dans la mort. Mais elle est le beau risque à courir de la vie. Au-delà du truisme qui accorde à la plante l'immobilité et à l'animal la motricité, Jonas découvre ainsi la vie animale sur le mode du risque et la vie végétale sur le mode de la sécurité ${ }^{11}$. La biologie philosophique étudie le végétal, l'animal et l'humain comme des formes de vie originales. Pour le végétal, le souci de la sécurité qui fait sa puissance peut dériver en tendance mortifère à l'inertie. Pour l'animal, le goût passionné pour la mobilité risque d'être exposé à l'instabilité. Pour l'humain, la puissance de sa technique dérive en acosmisme. Les formes pré-humaines du vivant, du végétal à l'animal, vivent ainsi «de brèves affirmations du sentiment de soi, d'un agir et d'un pâtir propres à des individus finis $»^{12}$. Elles vivent la satisfaction du plaisir et l'assurance de la sécurité mais aussi la possibilité du stress, de la peur et de la souffrance. La vitalité des vivants est plurielle et continue, démentant l'affirmation selon laquelle seul l'humain est formateur de monde. L'éthique de la responsabilité tirera les conséquences pratiques de cette conscience aiguë d'une appartenance qui solidarise humains et non humains.

7 On comprend alors pourquoi les classifications scolairement analytiques des éthiques de l'environnement en éthiques anthropocentrées, biocentrées ou écocentrées ne rendent pas avec justesse l'originalité du geste philosophique de Hans Jonas ${ }^{13}$. Il faut laisser retentir cette perplexité qui parait opposer éthique du respect et éthique de la responsabilité. Si la pensée de Hans Jonas partage avec ces éthiques l'idée que de la nature on peut faire un objet de responsabilité morale, elle s'en sépare par le traitement qu'elle en propose. Elle ne veut pas dissocier le souci écologique qui fait de la nature un objet de responsabilité d'une réflexion sur la philosophie de la culture et de l'être au monde. Aux éthiques de l'environnement il opposera une éthique pour la civilisation technologique. Sa cible est l'acosmisme d'une civilisation auquel remédier non en pensant moins l'homme - sa réduction au rang de parasite de la Terre - mais en le pensant mieux et davantage - le sens et la portée de ce que signifie « animal humain ». «Les possibilités apocalyptiques contenues dans la technologie moderne nous ont appris que l'exclusivisme anthropocentrique pourrait bien être un préjugé et qu'en tout cas il a besoin d'être réexaminé $»^{14}$.

Didactique, la distinction convenue entre les différentes éthiques de l'environnement fait de Hans Jonas un penseur anthropocentriste. Son éthique de la responsabilité et son nouvel impératif "Agis de façon que les effets de ton action soient compatibles avec la permanence d'une vie authentiquement humaine sur terre" feraient de l'homme la fin principale. Renforcerait cette idée, l'écart entre la préoccupation ontologique du Principe responsabilité qui se demande "pourquoi l'homme et la vie doivent-ils être? ? et le déploiement d'une éthique appliquée débouchant, moins sur une éthique de l'environnement que sur une éthique médicale. Pourtant, parce que Jonas ne dissocie pas la nature et l'histoire, l'analogie invitant à prendre soin de la nature et des hommes, sans pour autant confondre éthique médicale et éthique environnementale, prépare le passage du souci pour les humains vers celui des non humains. On pense à l'ouvrage Technique, médecine et éthique, dont le sous-titre est, rappelons-le, Pratique du principe responsabilité ${ }^{15}$. 
Notablement, son éthique médicale assume l'extension de la médecine clinique à la médecine des populations, cette dernière nouant santé et environnement. Elle examine les questions difficiles de l'épuisement des ressources naturelles en lien avec la charge démographique, ou celle de la misère de masse d'une humanité affamée. Plus encore, l'attention au phénomène de la vie maintient fortement le sens d'une appartenance des hommes à la nature. « En tant que puissance planétaire de premier ordre, il [l'homme] n'a plus le droit de penser à lui seul. [...] Bien comprise, l'intégration de l'existence de la richesse naturelle comme telle dans le bien humain, faisant ainsi de sa préservation un devoir, est suffisante pour dépasser le point de vue utilitaire et tout point de vue anthropocentrique $»^{16}$. Il convient donc d'apprendre à dialectiser ontologie de l'appartenance et éthique de la responsabilité dans des pratiques de la responsabilité instruites par une intelligence des contextes. Cette dialectique doit permettre d'échapper à l'acosmisme anthropologique. Il y aura ainsi dans les pratiques de la responsabilité, place pour une éthique médicale, une rare attention à l'animal et au végétal et une ouverture sur l'éthique environnementale. L'attention continue au phénomène de la vie se déploie en des expressions responsables discontinues en fonction des formes de vie qu'elle accompagne. L'axiologique principe responsabilité s'épelle dans des grammaires opératoires de la responsabilité. Il appelle une herméneutique des contextes où s'exerce une responsabilité en raison de l'importance de la médiation technique.

\section{2 - Un enjeu transversal : la question du mal}

9 Alors que l'éthique environnementale, et plus largement la philosophie de l'environnement, est devenue une discipline académique dans le contexte anglo-saxon, elle a longtemps été délaissée ou traitée avec méfiance par la philosophie dite «continentale». Celle-là valorise la nature, celle-ci ne cesse de penser la nature et l'histoire. On a parfois beau jeu d'en conclure que c'est bien là le signe manifeste de l'anthropocentrisme symptomatique de la tradition philosophique continentale. Sans doute est-il vrai que la réception de l'éthique environnementale en Europe, dans la seconde moitié $\mathrm{du} \mathrm{xx}^{\mathrm{e}}$ siècle, a été une réception tardive, occultée, peut-être ratée. L'Europe a du moins été suspicieuse à l'égard du discours eschatologique qui sous-tend le «retour à la Terre » et de l'impatiente réconciliation des hommes avec la nature qui l'anime qu'exalte parfois la valorisation de la wilderness. Elle l'a été, parce qu'elle demeure hantée et meurtrie par des violences totalitaires nourries de rédemptions eschatologiques (les religions séculières) dont l'écofascisme pourrait être une des résurgences. Cette réception ratée s'est ainsi enfermée dans une opposition artificielle entre l'antihumanisme d'une pensée de l'enracinement (idéologie du sol) et la mondialisation déterritorialisée triomphante d'une pensée de l'arrachement (idéologie du hors sol). En France, la controverse entre Michel Serres publiant Le Contrat naturel (1990) et Luc Ferry lui répliquant dans Le Nouvel Ordre écologique (1992) a été l'expression révélatrice de cette opposition, au moment même où l'on publiait la traduction française du Principe responsabilité (1990). Dans le cadre de cette réception, la philosophie de Hans Jonas n'a pas été épargnée par la critique. On a signalé que le ton apocalyptique de son éthique risquait de céder à une impatience eschatologique inattentive au travail de l'histoire. On a souligné que son heuristique de la peur portait une inquiétante et dangereuse dimension antidémocratique. On s'est alarmé du fait que son ontologie de la vie était suspecte d'être une métaphysique finaliste refoulée, antimoderne, sinon pré-moderne. Enfin on a observé 
que sa recherche philosophique paraissait décousue. Mais en dépit de toutes ces critiques, une observation s'impose. Il est difficile de faire de Jonas un philosophe continental ou un penseur d'éthique environnementale même s'il a vécu et écrit dans un contexte anglosaxon.

10 La position philosophique de Jonas, inactuelle en ce qu'elle noue éthique et ontologie, et ne renonce pas à la position d'un principe moral à portée universelle, résiste en portant une lancinante interrogation. Elle n'a cessé d'interroger comment les hommes, depuis le lointain de leur passé, ont tenté de répliquer au scandale du mal et à toutes les convulsions qu'il a pu connaître dans la nature et dans l'histoire. Ce défi du mal s'accroit encore lorsque ces hommes ne peuvent plus s'adosser sur l'assurance sécurisante d'un cosmos, livrés qu'ils sont, dans l'histoire, à un monde où déferlent les puissances mauvaises d'un appareil technoscientifique global et anonyme.

Qu'un spécialiste de la gnose en vienne à développer une éthique de la responsabilité susceptible de dépasser l'acosmisme de la modernité invite alors à faire une hypothèse. L'unité du questionnement de Jonas ne se tient-elle pas dans sa méditation sur le problème du mal et de la théodicée : mal cosmique dont l'homme est la victime avec la gnose ; mal de l'acosmisme avec la crise environnementale et sociale; mal que l'homme fait à l'homme dans une volonté de puissance affectant le concept de Dieu devenu Dieu sans puissance? En effet, trois ouvrages ponctuent le parcours dans les contrées du mal qu'explora Jonas. 1934-1954, La gnose et l'esprit de l'Antiquité tardive. I- Première partie: la gnose mythologique; II-Deuxième partie: de la mythologie à la philosophie mystique. 1979, Le Principe responsabilité. Une éthique pour la civilisation technologique. 1984, Le Concept de Dieu après Auschwitz. Une voix juive ${ }^{17}$. Cette méditation théorique continue sur le mal et l'appel à une réplique pratique au défi du mal, ne donnent-ils pas toute son épaisseur aux enjeux que mobilisent la responsabilité environnementale et la critique de l'acosmisme anthropologique? En effet, outre les aléas tragiques de l'histoire du $\mathrm{xx}^{\mathrm{e}}$ siècle qui ont traversé son histoire personnelle, Hans Jonas inscrit la préoccupation environnementale dans une mouvance phénoménologique instruite par une biologie philosophique et préparant un souci pratique, hanté par la question du mal. Cette hantise prend sous sa plume trois figures : la Gnose, le Prométhée déchaîné et Auschwitz ${ }^{18}$.

Mais comment passe-t-on de la gnose à la responsabilité ? Une anecdote rapporte qu'un étudiant américain rencontrant Paul Ricœur lui demanda s'il y avait un lien de parenté entre le Jonas des années trente connu pour un ouvrage sur le gnosticisme, La Gnose et l'esprit de l'Antiquité tardive, et celui des années 1970 connu pour ses travaux sur l'éthique, la responsabilité et l'éthique médicale. Sous l'ignorance de la question se cache un problème véritable. Quelle continuité conceptuelle entre le problème de la gnose et celui de la crise sociale et environnementale à laquelle réplique l'éthique pour la civilisation technologique? Peut-on penser l'unité d'un questionnement sous l'éclatement des objets abordés? Hans Jonas, relisant son parcours, y voyait trois étapes : « Le travail sur la gnose antique sous l'angle de l'analyse de l'existence; ensuite la rencontre avec les sciences de la nature sur le chemin vers une philosophie de l'organisme; enfin le tournant de la philosophie théorique vers la philosophie pratique - c'est-à-dire l'éthique - comme réponse au défi devenu de plus en plus pressant de la technique ${ }^{19}$. Cette périodisation factuelle est sous-tendue par une observation bien plus décisive. Il existe une analogie entre le nihilisme gnostique et le nihilisme moderne : la dualité de l'homme et du monde, même si le premier est exubérant et religieux et le second soupçonneux et athée. Cette analogie développe deux variantes d'un acosmisme hanté par l'énigme du mal. L'homme a 
été jeté. Il est plus précisément déjeté comme l'exprimera la littérature mandéenne, cette littérature sacrée liturgique ou divinatoire des $\mathrm{VII}^{\mathrm{e}}-\mathrm{IX}{ }^{\mathrm{e}}$ siècles qui se réfère à l'apocalypse de Saint Jean et qu'étudia Jonas. L'homme est perdu dans un monde qui lui est totalement étranger, que ce soit pour la gnose (de Marcion au manichéisme) ou pour l'existentialisme (de Pascal à Heidegger.) La « dévaluation existentialiste du concept de nature reflète la mise à nu spirituelle de cette nature aux mains de la science physique; elle a quelque chose de commun avec le mépris gnostique de la nature. Nulle philosophie ne s'est aussi peu souciée de la nature que l'existentialisme, aux yeux de qui elle n'a gardé aucune dignité $»^{20}$. Si l'on peut faire une lecture existentialiste du gnosticisme, une lecture gnostique de l'existentialisme est donc possible ${ }^{21}$.

Le passage de la gnose à la responsabilité se fait donc par une unité thématique - la méditation sur le mal - mais également par une méthode philosophique attentive aux existentiaux auxquels Jonas adjoindra ceux de génération future et de coprésence. Dans sa réflexion sur le mal, énigme théorique ou scandale pratique en appelant à une réplique éthique, Hans Jonas mobilisera une interprétation existentiale. Elle sera moins tournée vers l'être pour la mort que vers la vie, renouant les liens entre l'existant et les autres existants. Elle prendra ses distances avec l'être jeté qui sépare l'homme du reste du monde vécu. Mais cela se fera sans céder à la naïve réconciliation d'un principe espérance (cf. Marc Bloch) trop vite oublieux du désastre déferlant sur la nature et dans l'histoire. Jonas écrira ainsi qu' «après-guerre, [son] activité philosophique se plaça tout d'abord sous le signe d'un rejet de la philosophie heideggérienne de l'existence, à laquelle [il] opposai[t] sa philosophie de la vie $\aleph^{22}$. En effet, pointant une parenté profonde, à la hauteur d'un existential, entre l'être pour la mort et le souci éthique pour les générations futures, il dira que "la responsabilité comme telle n'est rien d'autre que le complément moral de la constitution ontologique de notre être temporel $»^{23}$.

L'attention à la question du mal est également prégnante dans l'impatience eschatologique qui nourrit la méditation jonassienne sur la catastrophe écologique. Ceci n'est pas sans rappeler les accents rédempteurs de la gnose valentinienne (Valentin est un chrétien gnostique du $\mathrm{II}^{\mathrm{e}}$ siècle aux écrits ésotériques déclaré hérétique) voulant sauver le moi spirituel de sa servitude cosmique. L'analogie s'arrêtera là cependant. Le drame gnostique concerne le vaste univers (même s'il est compris comme une petite cellule) alors que la crise environnementale se mobilise non sur l'univers mais sur la seule biosphère (même si le phénomène de la vie est une énigme cosmique). Le philosophe « catastrophiste » Jean-Pierre Dupuy note, avec humour, l'homonymie qui lie Hans Jonas le philosophe à la figure biblique de Jonas, le prophète de malheur ${ }^{24}$. Il y trouve l'occasion de réintroduire dans le débat contemporain une catégorie à laquelle nos réflexions éthiques sur le mal subi (la maladie comme mal physique) ou le mal commis (le mal que l'homme fait à l'homme comme mal moral) n'ont guère recours, à savoir le mal métaphysique. De fait, le penseur du catastrophisme éclairé a développé, à partir d'une réflexion sur le thème jonassien de l'heuristique de la peur, une analyse selon laquelle l'avenir nous juge au présent. Cette idée est mise au service de la responsabilité que convoque la crise sociale et environnementale occasionnée par le "Prométhée définitivement déchaîné $»^{25}$, figure mythique rendant compte de ce que, par ailleurs, Jonas appelle le panmécanisme. Lecteur de Jonas, Jean-Pierre Dupuy s'est concentré sur le problème suivant: "comment se fait-il que nous ne parvenions pas à croire ce que pourtant nous savons? ", à savoir que nous sommes en train d'entamer les héritages des générations à venir, leur empruntant un monde que nous devrions leur transmettre? 
L'explication de ce problème se trouverait, pour notre modernité tardive, dans un scandale du mal qui se serait déplacé en raison d'une inversion de la flèche du temps. Pour les philosophes des Lumières et les penseurs du progrès - Kant dans l'opuscule l'Idée d'une histoire universelle envisagée d'un point de vue cosmopolitique - le défi du mal était le suivant: sur le temps long de l'Histoire dans lequel l'humanité parvient progressivement à s'humaniser, le tribut que les générations les plus anciennes doivent payer est celui de se sacrifier pour le bien et l'amélioration morale de celles qui sont à venir. Or, inversement, le prophète de malheur, pourfend l'immobilisme qui tétanise l'action en raison de la préemption par les générations présentes des conditions d'un futur pour les générations à venir. Réplique à cette idée une compréhension renouvelée de la responsabilité. Là où classiquement elle renvoie à l'action déjà faite - l'imputabilité : être responsable de -, elle invitera à une responsabilité eu égard à ce qui n'est pas encore responsable pour... ou responsabilité sans pourquoi dira Levinas -, c'est-à-dire à une responsabilité sans réciprocité. Cette dernière s'épellera dans des pratiques de la responsabilité pensant le passage de l'éthique médicale à l'éthique environnementale. Jonas, en effet, ne craint pas d'adjoindre au principe responsabilité un versant appliqué concernant le mal de la maladie appréhendé par la technique médicale. Certes, on fera observer que l'éthique médicale a affaire au mal subi de la maladie. Mais, plus largement, la dimension publique de la médecine l'ouvre à une réflexion et prise en considération du malheur (de la famine comme question également sanitaire à la démographie planétaire galopante). Mais, dans tous les cas, le prophète dénonce l'oubli d'une évidence native. Nous avons oublié que «la Terre ne nous appartient pas mais que nous l'empruntons à nos enfants ", exprime le discours écologiste. Cet oubli n'est autre que l'oubli de la vie par une pensée aujourd'hui sous la domination ontologique de la mor ${ }^{26}$ comme Jonas s'applique à le démontrer. La temporalité prophétique tente donc de faire en sorte que le futur ait une actualité opératoire dans le présent. N'est-ce pas pourquoi Jonas cherchera dans le rôle dévolu à l'imagination, entendue comme une faculté du possible pratique, une anticipation du mal qui doit infléchir l'action? L'imagination n'est plus folle du logis mais faculté d'intensification du sens de notre responsabilité. Elle devient puissance, non plus tétanisante mais mobilisatrice en raison de sa force d'anticipation du mal à venir ${ }^{27}$.

La consistance d'une méditation sur le mal à partir de sa tonalité apocalyptique, l'importance reconnue à la peur comme un existential majeur mais aussi un rôle inédit dévolu à l'imagination sont donc des éléments décisifs dans la philosophie pratique de Jonas. La réflexion jonassienne sur l'acosmisme anthropologique à partir d'une méditation sur le mal, s'est donc faite tour à tour cosmologique, éthique puis finalement théologique. Il est frappant d'observer que le travail de Jonas, s'il débute par une réflexion sur l'acosmisme d'un Dieu inconnu absolument transcendant à l'histoire dans la religion gnostique, s'achève sur une théologie spéculative consacrée au silence d'un Dieu muet, à l'acosmisme non plus du Seigneur de l'histoire mais de la toute puissance technique des hommes mesurée à un Dieu sans puissance. Il sera passé entre temps par une biologie philosophique qui enrôle toutes les formes du vivant (végétale, animale ou humaine) dans une lutte du métabolisme pour la vie et un combat dans la vaste histoire du mal. Le risque du mal débute d'ailleurs, quoique ce soit inattendu, avec le végétal, cette créature du silence. Il se poursuit avec l'animal, cette créature de la faim puisque l'apparition de la vie suppose déjà celle de la mort. Il s'élargit avec l'homme. Jonas aura développé, pour montrer ce dernier point, une analyse immanente consacrée à ce rôle de titan qu'est le prométhéisme, celui de l'économie et de la technique moderne, auquel doit répondre une conception de la responsabilité renouant à nouveaux frais l'homme avec les 
hommes et les non-humains, mais sans sans espérance facile. À la puissance technique indéfinie des hommes doit répliquer une responsabilité infinie pour les vulnérables. "Le principe responsabilité qui, selon Hans Jonas, répond à cette douleur [celle pour laquelle toute philosophie ne peut plus se construire en tournant le dos à l'agonie des hommes et du monde] se désolidarise en effet de toute thèse réconciliatrice, il affirme simplement qu'il faut vivre et penser avec la certitude de la proximité toujours là du mal et exiger de soi ce qui permet de l'empêcher, non de faire semblant de le réparer lorsqu'il se fait tard et que la plainte a cessé $»^{28}$. Il est donc un triple drame que Jonas n'a eu de cesse de méditer. Drame d'un mal cosmique dont l'homme est l'objet, la victime et le centre. Drame du mal écologique dont l'homme, et avec lui tous les vivants, est le sujet tout autant que l'objet puisqu'il s'agit d'un mal physique qui s'origine dans un mal moral. Drame du mal génocidaire dont l'homme est sujet-objet exigeant de reformuler notre compréhension des attributs divins, et de renoncer au tyrannique désir de toute puissance tout en laissant l'homme devant sa responsabilité. Ni l'homme gnostique, ni l'homme existentialiste, c'est la figure de Job que retient finalement Jonas pour subordonner le déferlement du mal technique et politique à la responsabilité morale. Mais il s'agit d'un Job renonçant à l'idée d'un Dieu seigneur de l'histoire. Cette figure de Job conduit Jonas à une solution pratique immanente sur fond de tragique : le principe responsabilité comme remède pratique aux incertitudes du temps. En effet, observons que Jonas ne retiendra pas, cela aurait pu donner une toute autre perspective développée aujourd'hui d'ailleurs par certaines théologies de la création, l'étonnant final du livre de Job (Job, 38-41). Ce final qui n'évoque en rien un quelconque anthropocentrisme ou une domination de l'homme sur la nature, met en évidence les limites des humains. Ils se révèlent des maîtres bien modestes, souvent oublieux des forces de la nature qui échappent structurellement et profondément à leur emprise. Ce final dépasse l'acosmisme anthropologique par la célébration d'une exigence de plénitude, celle d'une appartenance de l'homme à l'univers qui ne méconnaît pas le tragique de l'histoire. Mais Hans Jonas n'a pas retenu cette voie.

\section{3 - Surmonter éthiquement l'acosmisme anthropologique}

Pour conclure, nous observerons que la coprésence, la biologie philosophique et le concept de "génération future » sont trois leviers décisifs sur lesquels s'est appuyé Jonas pour dépasser l'acosmisme anthropologique. Ce faisant, il n'a pas craint de lier ensemble, par le biais de sa biologie philosophique, une phénoménologie de l'appartenance du vivant humain au monde de la vie et une herméneutique des cultures, notamment de la civilisation technologique, tout en en appelant à une éthique pour le futur.

Hans Jonas envisage la civilisation technologique, et la crise écologique qui l'accompagne, comme un phénomène spirituel, différent, mais non moins majeur que le phénomène gnostique. «Il y a un gnosticisme latent de l'esprit moderne, qui l'apparente, toutes différences mises à part, au dualisme de l'ancienne gnose, au moins pour autant que la tentation extrémiste existe de part en part $»^{29}$. Cette civilisation technologique déploie une compréhension du temps porteuse d'une eschatologie sécularisée (le progrès et avec lui le "principe espérance " dérivant avec la modernité tardive en accélération); une conception de la nature neutralisée sous l'angle de la valeur; la disparition d'un sacré fondateur. Y répliquera, non plus théoriquement mais pratiquement, une conception 
renouvelée de la responsabilité éthique assise sur l'attention aux conséquences imprévisibles de nos activités techniques sur le monde naturel et les relations interhumaines. Ce principe responsabilité ambitionne de dépasser l'acosmisme anthropologique. Il repose sur une définition du statut ontologique de l'homme, sur une herméneutique du vivant et enfin sur un connecteur temporel qui lie ensemble la nature et l'histoire : les générations futures.

La crise écologique contemporaine tient à l'acosmisme d'un dualisme ontologique qui a réduit la nature au rang de carrière à exploiter. Repenser ce que les anciens n'hésitaient pas à appeler l'animal raisonnable pourrait contribuer à la résorber. Or, pour Hans Jonas, l'extrême élévation acosmique de l'homme va de pair avec une extrême aliénation cosmique de l'homme jeté au monde. L'acosmisme anthropologique de l'existentialisme encourage une pensée de l'exil, ou de la séparation radicale de l'existant parmi les vivants plutôt qu'il invite à une pensée de la coprésence. Il prépare ainsi, sur fond de séparation, une domination sans limites de la nature. Dans une importante note ${ }^{30}$ consacrée à l'interprétation heideggérienne du statut de l'animal rationnel, Hans Jonas observe que ce statut peut certes s'interpréter comme l'installation sur une échelle des êtres qui rabaisserait l'homme et l'aliénerait en le tirant vers l'animalité - l'interprétation existentielle - mais qu'elle peut aussi se comprendre comme l'installation de l'humain dans le vaste déploiement des êtres animés. L'animal rationnel s'en trouve de la sorte moins rabaissé que situé. Il s'envisage non comme l'être de l'antinature mais comme celui qui est engagé dans un régime de coprésence. Ce dernier ouvre la perspective d'un dépassement de l'acosmisme, sortant de l'alternative entre exister ou être aliéné, entre situation d'existence et être à disposition, en se concentrant sur ce que donne ou exprime la nature. "Après tout, en dehors du "présent » existentiel, celui du moment, il y a le présent des choses. La coprésence avec elles ne procure-t-elle pas un "présent » d'une espèce différente? $»^{31}$ C'est cette présence nue, fragile et muette de la vie, située en amont de tout ce qui se tient à disposition pour être exploité, qui constituera le cœur de la responsabilité. Au-delà de la situation existentielle ou de l'inscription matérielle, la coprésence manifeste un vivant livré, vulnérable, tout entier exposé. La coprésence est l'envers ontologique du dualisme acosmique. Tous les vivants ont en partage cette vulnérabilité, l'homme compris, mais l'homme en sera responsable parce qu'il en est conscient.

19 À cette attention ontologique, Hans Jonas adjoint une biologie philosophique qui, sans céder à une métaphysique de la vie, accepte de reconnaître que la question de la téléologie n'est pas ridicule mais sensée. Il élabore une herméneutique de la nature redistribuant les relations scolairement distinguées entre sciences de la nature explicatives et sciences humaines compréhensives. Pour Jonas, il y a de la compréhension engagée dans l'explication des phénomènes naturels. Elle est liée à une redéfinition attachée à la subjectivité (distincte de l'ipséité) de tout vivant qui attend que l'on comprenne des formes de vie. Au-delà de l'opposition entre mécanisme et finalisme, entre matérialisme et idéalisme, la vie est une unité équivoque. Cette équivocité ne saurait être épuisée dans la seule description explicative de processus mécanistes, pas plus que mystérieusement voilée dans un finalisme psychique. "Expliquer ou comprendre la nature ce n'est pas la même chose $»^{32}$. On doit en conclure que la biologie n'est pas qu'une physique de la nature mais une herméneutique de la nature expressive, attentive aux formes de la vie. Elle doit comprendre une solidarisation fondamentale et croissante engagée dans le déploiement de la vie qui engage une solidarité éthique. On 
peut penser ainsi l'éthique à partir de l'inscription de l'homme dans la nature sans revenir au finalisme de l'antiquité classique, ni céder à la conception romantique d'une nature réenchantée. Se substituant à l'histoire naturelle, la biologie philosophique pour laquelle le fait qu'il y ait de la vie plutôt que pas n'est pas indifférent, inaugure le monde de la vie comme monde de la valeur. La vie qui reconnaît la vie dans la sécurité vitale de la plante, ou le désir de mobilité de l'animal n'est pas neutre éthiquement. Elle préfigure l'unité d'une aventure de la vie qui prend conscience d'elle-même jusqu'à s'expliciter dans la responsabilité éthique. En ce sens la responsabilité est sur le plan réfléchi de l'éthique ce qu'est l'épreuve sentie d'une coprésence des vivants sur le plan biologique. "La question que l'homme est à lui-même n'est que l'écho lointain d'une question originaire que la vie s'est posée à elle-même en se lançant dans l'aventure de l'évolution. En s'affirmant chaque fois avec plus de risque, elle se donne à apprécier avec toujours davantage de liberté $»^{33}$. La biologie philosophique, entendue comme une herméneutique de la nature, établit la médiation entre une ontologie de la coprésence et une éthique de la responsabilité en démontrant que la nature et les diverses formes du vivant ne sont pas des matériaux éthiquement neutres. Stratégiquement, cette biologie philosophique sert donc de pivot entre la réfutation ontologique de l'acosmique et la réplique éthique à ce dernier. S'il existe des pratiques diverses de la responsabilité, diverses éthiques appliquées comme on le dirait aujourd'hui, c'est en raison du fait que la biologie philosophique nous rend attentive à des formes bien singularisées de la vie. Végétale, animale ou humaine, ces valorisations vitales à chaque fois singulières en appellent à une forme d'attention éthique non moins singulière. La force de la biologie philosophique est donc de faire apparaitre la consistance de médiations contextualisantes qui permettront de distinguer la biologie, la médecine ou l'écologie de la conservation. Ces médiations servent de propédeutique à des pratiques plurielles de la responsabilité. Herméneutique du vivant non tronquée par la modélisation mécaniste, la biologie philosophique prolonge le questionnement sur les vivants et l'humain par une information nourrie des sciences contemporaines, précise et démystifiante. Ce faisant, elle embarque les vivants sous la continuité des lois de la biologie qu'elle interprète comme une vaste histoire de résistance à la mort et dont l'éthique est l'expression explicitée et consciente. On en tirera une conséquence importante. C'est comme vivant que l'homme est chargé éthiquement de prendre soin de la vie en général et de la pluralité de ses formes.

Enfin, Le principe responsabilité articule régime ontologique de co-présence, diversité des formes du vivant et responsabilité éthique en reformulant l'impératif catégorique. Le concept de "générations futures " en est le pivot. Hans Jonas propose, en raison de la spécificité des pouvoirs techniques contemporains, différentes formulations de l'impératif catégorique renouvelant notre compréhension du devoir. Le fait d'en pouvoir plus en appelle à en devoir davantage. C'est pourquoi il articule responsabilité synchronique - les générations présentes - et diachronique - les générations futures. "Agis de façon que les effets de ton action soient compatibles avec la permanence d'une vie authentiquement humaine sur terre » ou bien encore "Inclus dans ton choix actuel l'intégrité future de l'homme comme objet secondaire de ton vouloir $\aleph^{34}$. Il ne s'agit pas là d'une conception inflationniste et démotivante de la responsabilité. Le caractère illimité de la responsabilité sur le long terme parait en appeler à une forme d'infinitisation qui déresponsabilise. On peut se demander en effet où s'arrête le futur d'une éthique du futur? Mais la force de la proposition de Jonas réside dans le concept de génération future, non pour sur-responsabiliser mais pour penser à nouveaux frais la responsabilité 
à partir d'un appel de la vulnérabilité. Elargissant le temps de la responsabilité du temps des contemporains au temps des successeurs, il permet, sur le plan éthique, de dépasser l'acosmisme. Paul Ricœur, dans Temps et récit $t^{35}$, proposait le concept de connecteur temporel afin d'articuler temps individuel et temps cosmique. Stratégiquement ce concept servait à sortir de l'aporie opposant le temps phénoménologiquement vécu et le temps universel objectif, c'est-à-dire à éviter le risque d'acosmisme. Or, le concept de génération future ne peut-il pas lui aussi être envisagé comme un connecteur temporel dans sa portée existentiale, donnant corps à la coprésence évoquée ci-dessus ? D'une part, il solidarise le temps individuel et le temps social par l'idée d'une suite des générations et pas seulement d'une succession de générations indifférentes les unes aux autres. D'autre part, le futur convoqué dans l'idée de génération future connecte le temps social au temps cosmique, articulant le temps de l'action des hommes avec le grand temps du monde par un être affecté par le futur. Ainsi, noue-t-il de façon immémoriale la responsabilité pour ce qui est et ce qui doit être.

En parlant de coprésence, de biosphère, de génération future, tout en plaçant ses analyses sous une méditation sur le scandale du mal, Hans Jonas a donc lesté le souci environnemental d'une dimension d'historialité attentive à notre être affecté par la relation au monde de la vie qui constitue une énigme originaire: celle de notre appartenance au phénomène de la vie. Il le leste également d'un coefficient d'historicité présent dans les communautés historiques car c'est au sein des cultures, parfois acosmiques, que les hommes apprennent à se comprendre comme étant de la nature. La crise environnementale engendrée par l'acosmisme de la civilisation technologique questionne cette dernière, demandant ce que signifie métaphysiquement, scientifiquement et pratiquement la vulnérabilité de la vie tout entière remise en notre pouvoir et en notre devoir. Être en prise avec la vie ne cesse d'interroger notre emprise sur la vie.

\section{NOTES}

1. Hans Jonas, «Gnosticisme, existentialisme et nihilisme » dans La Religion gnostique, Le message du Dieu étranger et les débuts du christianisme (1958), trad. Louis Evrard, Paris, Flammarion, 1978, p. 423.

2. Ibidem, p. 442.

3. Le principe responsabilité, Une éthique pour la civilisation technologique (1979), trad. fr. et présentation J. Greisch, Paris, Cerf, 1990, p. 24.

4. Ibidem, p. 27.

5. Paul Ricœur, Soi-même comme un autre, Paris, Seuil, 1990, p. 178.

6. «La responsabilité prioritaire pour le long terme est sans doute d'empêcher que la planète entière, la Terre, notre étroit vaisseau spatial, ne devienne un... bateau de sauvetage désespéré et déshumanisé ", Hans Jonas, L'art médical et la responsabilité humaine, trad. de l'allemand Technik, Medizin und Ethik (1985) par Eric Pommier, Paris, Cerf, 2012, p. 56.

7. Voir E. Husserl, «L'arché originaire Terre ne se meut pas", trad. D. Franck, Paris, Revue Philosophie, Éditions de minuit, 1989. Husserl fidèle à son programme théorique porte moins le 
souci de fonder une éthique environnementale que la recherche d'une couche eidétique fondamentale : la Terre comme lieu de notre séjour.

8. Martin Heidegger, Lettre sur l'humanisme (1946), trad. Roger Munier, Paris, Aubier, 1957.

9. La biosphère (1926), Paris, Seuil, « Points », 2002.

10. L'art médical et la responsabilité humaine, op. cit., p. 40.

11. Le phénomène de la vie. Vers une biologie philosophique, trad. D. Lories, Bruxelles, De Boeck Université, 2001.

12. Hans Jonas, Le concept de Dieu après Auschwitz (1984), trad. Ph. Ivernel, Paris, Payot et Rivages, 1994, p. 17.

13. À titre d'exemple, dans son ouvrage très clair par ailleurs, Gérald Hess, lorsqu'il présente la philosophie de Hans Jonas, fait de ce dernier un écocentriste naturaliste, dans une perspective pragmatique. Mais il achève sa présentation en lui reprochant finalement de défendre, en dépit de son soubassement ontologique, un point de vue anthropocentriste. Voir Les éthiques de la nature, Paris, PUF, 2013, pp. 282-292 et p. 292.

14. Le principe responsabilité, op. cit., p. 72.

15. Technik, Medizin und Ethik (1985).

16. L'art médical et la responsabilité humaine, op. cit., p. 27.

17. Quant à la théologie juive aujourd'hui, elle doit se confronter avec la réalité et la réussite du mal délibéré, bien plus qu'avec les épreuves de l'aveugle causalité naturelle - Auschwitz et non pas le tremblement de terre de Lisbonne. Hans Jonas, Le concept de Dieu après Auschwitz, op. cit., pp. 36-37.

18. On pense à Hiroshima, autre figure du mal dans le télémeurtre qu'étudiera son condisciple, Günter Anders.

19. Le phénomène de la vie. Vers une biologie philosophique (1966), trad. D. Lories, Bruxelles, De Boeck Université, 2001, p. 218.

20. Hans Jonas, « Gnosticisme, existentialisme et nihilisme », op. cit. p. 439.

21. «Nous avons été jeté. [...] La force de choc de cette image possède elle-même une valeur symbolique dans le récit gnostique de l'existence humaine. Il y aurait grand intérêt à comparer l'emploi qu'en fait le gnosticisme et l'emploi qui en est fait dans une très récente analyse philosophique de l'existence, celle de Martin Heidegger. ", La Religion gnostique, op. cit., p. 92.

22. H. Jonas, «Adieu à Heidegger» in Souvenirs (2003), trad. fr. S. Cornille et P. Ivernel, Paris, Payot et Rivages, 2005, p. 225.

23. Le principe responsabilité, op. cit, p. 210.

24. «Avouerai-je enfin que le dernier trait qui me séduit chez Jonas, c'est son nom? Il partage celui-ci avec un autre prophète de malheur qui lui est antérieur de près de vingt-huit siècles. Sans tomber dans le mysticisme, je ne peux m'empêcher d'y voir comme un prodigieux clin d'œil $\mathrm{du}$ destin. Les théoriciens de la précaution aimeraient bien jeter par-dessus bord le Jonas qui les embarrasse, comme des marins étrangers s'y résolurent dans le cas de son lointain ancêtre.", Jean-Pierre Dupuy, Le catastrophisme éclairé. Quand l'impossible est certain. Paris, Le Seuil, 2006, pp. 97-98.

25. Le principe responsabilité, op. cit., p. 13.

26. Le phénomène de la vie, op. cit., p. 23.

27. Dans notre situation historique, «ce qui doit être craint n'a précisément pas encore été éprouvé. [...] Ici le malum imaginé doit donc assumer le rôle du malum éprouvé [...] se procurer cette représentation par une pensée tournée vers l'avenir devient la première obligation. ", Hans Jonas, Le principe responsabilité, op. cit., p. 50.

28. Catherine Chalier, «Dieu sans puissance », postface dans Hans Jonas, Le concept de Dieu après Auschwitz, op. cit., pp. 68-69.

29. Jean Greisch, «Présentation » au Principe responsabilité, op. cit., p. 11.

30. La Religion gnostique, op. cit., note 2, p. 434.

31. La Religion gnostique, op. cit., p. 439. 
32. Le principe responsabilité, op. cit., p. 105.

33. Eric Pommier, Ontologie de la vie et éthique de la responsabilité selon Hans Jonas, Paris, Vrin, 2013, p. 85 .

34. Le principe responsabilité, op. cit., pp. 30-31.

35. Temps et récit, III, Le temps raconté (1985), Paris, Seuil, "Points", 2005, voir le chapitre 1 «Entre le temps vécu et le temps universel : le temps historique », pp. 189-228. 